\title{
A UNIFIED APPROACH TO GENERALIZED INVERSES OF LINEAR OPERATORS: II. EXTREMAL AND PROXIMAL PROPERTIES
}

\author{
BY M. Z. NASHED AND G. F. VOTRUBA
}

Communicated by Robert Bartle, December 12, 1973

1. Introduction. This note is a sequel to [5]; the notations are the same. An important property of the Moore-Penrose inverse $T^{\dagger}$ of a matrix, or a bounded linear operator, or a densely-defined closed linear operator $T$ on a Hilbert space is the relation of $T^{\dagger}$ to extremal solutions of the equation $T u=y$ (see [2], [4], [7]). We develop proximal properties of generalized inverses in normed spaces within the general setting of [5] and demonstrate their relations to extremal, minimal, and best approximate solutions.

2. Preliminaries. Let $N$ be a normed linear space. Let $\operatorname{sp} A$ denote the span of a set $A$ and $d(x, A)$ the distance from $x$ to $A \subset N$. The following definition of orthogonality is used: $x \perp y$ means $d(x, \operatorname{sp}\{y\})=\|x\|$, and $B \perp A$ means $d(x, A)=\|x\|$ for each $x \in B$. Note that this orthogonality is not a symmetric relation. Let $M$ be a subspace of $N$ which has a topological complement, and consider the affine manifold $\mathscr{P}_{M}=\{P \in \mathscr{L}(N)$ : $P^{2}=P$ and $\left.\mathscr{R}(P)=M\right\}$.

Proposition 1. Let $P_{0} \in \mathscr{P}_{M}$. The following statements are equivalent:

(a) $P_{0}$ is the nearest point in $\mathscr{P}_{M}$ to $I$, and $\left\|I-P_{0}\right\|=1$.

(b) $\mathscr{N}\left(P_{0}\right) \perp \mathscr{R}\left(P_{0}\right)$.

(c) For each $x \in N, P_{0} x$ is the nearest point in $M$ to $x$.

If there exists a $P_{0} \in \mathscr{P}_{M}$ such that any (and hence all) of the statements in Proposition 1 hold, we say that $M$ is an orthogonally-complemented subspace of $N$. We emphasize that if $M=\mathscr{R}\left(P_{0}\right)$ is orthogonally complemented by $\mathscr{N}\left(P_{0}\right)$, it is not necessarily true that $\mathscr{N}\left(P_{0}\right)$ is orthogonally complemented by $\mathscr{R}\left(P_{0}\right)$. Also, orthogonal complements are not necessarily unique. Hilbert spaces are an exceptional case; if $M$ is a closed

AMS (MOS) subject classifications (1970). Primary 47A50, 47A99, 41A50; Secondary $54 \mathrm{C} 60,54 \mathrm{C} 65$.

Key words and phrases. Extremal solutions, metric generalized inverse, linear operators, orthogonally-complemented normed subspaces, orthogonal partial inverses, metric selections. 
subspace of a Hilbert space, then $M^{\perp}$ is the unique orthogonal complement of $M$ in the above sense, and also $M^{\perp}$ has $M$ as an orthogonal complement. In this case the corresponding projectors will be selfadjoint. Finally we remark that in a normed linear space every one-dimensional subspace is an orthogonal complement for some hyperplane, for let $S=\operatorname{sp}\{e\}$, $\|e\|=1$. Take (by the Hahn-Banach theorem) $f \in N^{*}$ with $\|f\|=1$ and $f(e)=1$. Define $P$ by $P x=x-f(x) e$. Then $\mathscr{R}(P)=\mathscr{N}(f)$ and $\|I-P\|=1$. In general, in normed linear spaces, orthogonally-complemented subspaces are rare.

3. Main results. Let $\Lambda(V, W)$ denote the space of all linear maps from a vector space $V$ into a vector space $W$. If $T \in \Lambda\left(V, N_{2}\right)$ where $N_{2}$ is a normed space, then $v_{0} \in V$ is called an extremal solution of the equation $T v=y$ if $v=v_{0}$ minimizes $\|T v-y\|$. If $V=N_{1}$ is also a normed space, then an extremal solution of minimal norm is called a best approximate solution or a least extremal solution. The equation $T v=y$ need not have an extremal solution for each $y$, and the existence of an extremal solution does not imply the existence of a best approximate solution.

Definition 2. Let $N_{2}$ be a normed linear space, and let $T \in \Lambda\left(V, N_{2}\right)$. If $U=T_{r, Q}^{\dagger}$ is a R-T.P.I. of $T$ where $\|I-Q\|=1$, we call $U$ a rightorthogonal partial inverse (abbreviated as R-O.P.I.) of $T$. On the other hand, if $\mathscr{D}(T)=V$ is contained in a normed linear space $N_{1}, \mathscr{R}(T)$ is in a (algebraic) vector space $W$, and $U=T_{l, P}^{\dagger}$ is a L-T.P.I. of $T$, where $\|I-P\|=1$, we call $U$ a left-orthogonal partial inverse (L-O.P.I.). If $U$ is both a L-O.P.I. and a R-O.P.I. we call $U$ an orthogonal partial inverse (O.P.I.) of $T$.

Note that by Proposition 1, the existence of a R-O.P.I. implies that $\overline{\mathscr{R}(T)}$ is an orthogonally-complemented subspace of $N_{2}$. Also, the existence of a L-O.P.I. implies that $\mathscr{C}_{P}(T) \perp \mathscr{N}(T)$ since $\mathscr{N}(P) \perp \mathscr{R}(P)$ by Proposition 1 and since $\mathscr{N}(T) \subset \mathscr{R}(P)$ and $\mathscr{C}_{P}(T) \subset \mathscr{N}(P)$ by [5, Theorem 2.4, Definition 2.2]. Thus, in general, the existence of an O.P.I. is a rare phenomenon in normed spaces without inner products.

If $\mathrm{N}_{2}=\mathrm{H}_{2}$ is a Hilbert space, there is a unique $Q$ with range $\overline{\mathscr{R}(T)}$ satisfying $\|I-Q\|=1$. Thus for $T \in \Lambda\left(V, H_{2}\right)$, a R-O.P.I. always exists. If $N_{1}=H_{1}$ is also a Hilbert space, then $T$ has an O.P.I. if and only if $T$ is decomposable with respect to a selfadjoint projector $P$ in the sense of [5, Definition 2.2].

The importance of right-orthogonal, left-orthogonal, and orthogonal partial inverses lies in their connection with the existence of extremal solutions, minimal solutions, and best approximate solutions of the equation

$$
T x=y \text {. }
$$


THEOREM 3. If $U$ is a right-orthogonal partial inverse of $T \in \Lambda\left(V, N_{2}\right)$, then for $y \in \mathscr{D}(U)$ the equation (1) has $v=U y$ as an extremal solution. If, in addition, nearest points from $\overline{\mathscr{R}(T)}$ are unique (in particular if $N_{2}$ is strictly convex), then the existence of an extremal solution implies that $y \in \mathscr{D}(U)=\mathscr{R}(T) \oplus \mathscr{N}(Q)$.

THEOREM 4. If $U$ is a left-orthogonal partial inverse of $T$ and the equation (1) has a solution, then Uy is a (not-necessarily unique) solution of minimal norm.

THEOREM 5. If $U$ is an orthogonal partial inverse of $T$, and if in $N_{2}$ nearest points from $\overline{\mathscr{R}(T)}$ are unique, then for all $y \in \mathscr{D}(U), x=U T U y=$ $T_{P, Q}^{\dagger} y$ is a (not-necessarily unique) best approximate solution of (1).

Corollary 6. Let $H_{1}$ and $H_{2}$ be Hilbert spaces and let $T: \mathscr{D}(T) \subset$ $H_{1} \rightarrow H_{2}$. Then (1) has an extremal solution if and only if the orthogonal projection of $y$ onto $\overline{\mathscr{R}(T)}$ lies in $\mathscr{R}(T)$. In this case (1) has a (unique) best approximate solution if and only if the orthogonal projection of any (every) extremal solution onto $\overline{\mathscr{N}(T)}$ lies in $\mathscr{N}(T)$. If $T$ has an orthogonal partial inverse, then (1) has a unique best approximate solution whenever it has an extremal solution.

This corollary basically contains the results given in Erdelyi and Ben-Israel $[2, \S 2]$. For matrices, it is well known that $\|A x-b\|_{2}$ is minimized by $x=U b$ if $U$ satisfies $A U A=A$ and $A U$ is selfadjoint (see e.g. Rao and Mitra [8]). This is a special case of Theorem 3. Also if $A x=b$ has a solution, then the unique solution of smallest norm is $x=U b$ where $A U A=A$ and $U A$ is selfadjoint. This follows as a special case of Theorem 4 plus the use of strict convexity which gives uniqueness. Finally the wellknown results for a bounded or densely-defined closed linear operator on a Hilbert space with closed or nonclosed range (see, e.g., [4]) are also corollaries.

In view of Theorem 5, which gives the existence of best approximate solutions, it is useful to consider another kind of generalized inverse.

Definition 7. Let $T \in \Lambda\left(N_{1}, N_{2}\right)$, and consider a $y \in N_{2}$ such that $T x=y$ has a best approximate solution in $N_{1}$. We define

$$
T^{\partial}(y)=\left\{x \in N_{1} \mid x \text { is a best approximate solution to } T x=y\right\}
$$

and call the set-valued mapping $y \rightarrow T^{\partial}(y)$ the metric generalized inverse. Here $\mathscr{D}\left(T^{\partial}\right)=\left\{y \in N_{2} \mid T x=y\right.$ has a best approximate solution in $\left.N_{1}\right\}$. A (in general nonlinear) function $T^{\sigma}(y) \in T^{\partial}(y)$ is called a selection for the metric generalized inverse.

From Theorem 3 we see that if $T$ has a right-orthogonal partial inverse and if nearest points from $\overline{\mathscr{R}(T)}$ are unique, then $\mathscr{D}\left(T^{\partial}\right) \subset \mathscr{R}(T) \oplus \mathscr{N}(Q)$. 
If in addition $T$ has an orthogonal partial inverse $U$, then $\mathscr{D}\left(T^{\partial}\right)=\mathscr{D}(U)=$ $\mathscr{R}(T) \oplus \mathscr{N}(Q)$ and $T_{P, Q}^{\dagger}=U T U$ is a linear selection for $T^{\partial}$.

4. Remarks and special cases of the metric generalized inverse. (i) Holmes [3] defines the generalized inverse of $T \in \mathscr{L}\left(N_{1}, N_{2}\right)$ to be $T^{\partial}$ in the case where $T^{\partial}(y)$ is always a singleton and gives some conditions which imply that $T^{\partial}$ is densely defined and/or continuous. He uses this approach to obtain the (linear) generalized inverse in the Hilbert space case.

(ii) Newman and Odell [6] have studied $T^{\partial}$ in the finite-dimensional case where the norms are strictly convex. In this case, there is always a unique best approximate solution of $L x=y$ given by $B=(I-F) M E$ where $M$ is any partial inverse of $L, E$ is the nearest point map onto $\mathscr{R}(A)$, and $F$ is the nearest point map onto $\mathscr{N}(A)$. Some of the results of Erdelsky [1] are also subsumed by our theorems.

(iii) Erdelyi and Ben-Israel [2] considered the case of an arbitrary linear mapping with domain in a Hilbert space $H_{1}$ and range in a Hilbert space $H_{2}$. In this case $T^{\partial}(y)$ is always a singleton and $T^{\partial}$ is a linear map. They used the name $g$-inverse. Here $\mathscr{D}\left(T^{\partial}\right)=T(\mathscr{C}) \oplus \mathscr{R}(T)^{\perp}$ where $\mathscr{C}=$ $\mathscr{D}(T) \cap \mathscr{N}(T)^{\perp}$, and $\mathscr{R}(T) \notin \mathscr{D}\left(T^{\partial}\right)$ in general. Let

$$
\tilde{T}=T \mid \mathscr{D}_{0} \quad \text { where } \mathscr{D}_{0}=\mathscr{N}(T) \oplus \mathscr{C} \text {. }
$$

Then $\tilde{T}$ has an orthogonal generalized inverse $\tilde{T}^{\dagger}$ in the sense of Definition 2 with domain $T(\mathscr{C}) \oplus T(\mathscr{C})^{\perp}$, and the $g$-inverse turns out to be $T^{\partial}=$ $\tilde{T}^{\dagger} \mid \mathscr{D}\left(T^{\partial}\right)$. If $T$ is decomposable with respect to a selfadjoint projector, then $T^{\partial}=T^{\dagger}$; see Corollary 6 .

(iv) The problem of obtaining selections with nice properties for the metric generalized inverse of linear and nonlinear operators merits study.

The proofs and some additional results will be published elsewhere.

\section{REFERENCES}

1. P. J. Erdelsky, Projections in a normed linear space and a generalization of the pseudoinverse, Doctoral Dissertation, California Institute of Technology, Pasadena, Calif., 1969.

2. I. Erdelyi and A. Ben-Israel, Extremal solutions of linear equations and generalized inversion between Hilbert spaces, J. Math. Anal. Appl. 39 (1972), 298-313.

3. R. B. Holmes, $A$ course on optimization and best approximation, Lecture Notes in Math., vol. 257, Springer-Verlag, Berlin, 1972.

4. M. Z. Nashed, Generalized inverses, normal solvability, and iteration for singular operator equations, Nonlinear Functional Anal. and Appl. (Proc. Advanced Sem., Math. Res. Center, Univ. of Wisconsin, Madison, Wis., 1970), Academic Press, New York, 1971, pp. 311-359. MR 43 \#1003.

5. M. Z. Nashed and G. F. Votruba, A unified approach to generalized inverses of linear operators: I. Algebraic, topological, and projectional properties, Bull. Amer. Math. Soc. 80 (1974), 825-830. 
6. T. G. Newman and P. L. Odell, On the concept of a p-q generalized inverse of a matrix, SIAM J. Appl. Math. 17 (1969), 520-525. MR 41 \#220.

7. R. Penrose, On best approximate solutions of linear matrix equations, Proc. Cambridge Philos. Soc. 52 (1956), 17-19. MR 17, 536.

8. C. R. Rao and S. K. Mitra, Generalized inverse of matrices and its applications, Wiley, New York, 1971.

School of Mathematics, Georgia Institute of Technology, Atlanta, Georgia 30332

Department of Mathematics, University of Montana, Missoula, Montana 59801 